\title{
Remoción del Colorante Rojo Allura en Solución Acuosa utilizando Carbones Activados obtenidos de Desechos Agrícolas
}

\author{
Adolfo E. Ensuncho, Nathaly Milanés y Juana R. Robles \\ Universidad de Córdoba, Departamento de Química, Cra 6 N76-103, Córdoba-Colombia. \\ (e-mail: adem9abril@hotmail.com;namios20@gmail.com;juanarobles2003@yahoo.com)
}

Recibido Jul. 10, 2014; Aceptado Sep. 23, 2014; Versión final recibida Nov. 11, 2014

\begin{abstract}
Resumen
Se estudió la remoción del colorante rojo allura con diferentes tipos de carbón activado, obtenido a partir varias fuentes de desechos agrícolas. Los carbones se caracterizaron mediante espectroscopia infrarroja, difracción de rayos $\mathrm{X}$ y microscopía electrónica de barrido, obteniéndose resultados típicos de materiales carbonáceos. Las áreas superficiales de los carbones activados, se determinaron mediante isotermas de nitrógeno. De igual manera, se evaluaron las isotermas de remoción del colorante teniendo en cuenta, el efecto del tiempo de contacto, $\mathrm{pH}$, la concentración inicial y la cantidad de adsorbente. El carbón tuza de maíz, mostró mayor porcentaje de remoción del colorante con respecto a los carbones cáscara de coco y cascarilla de arroz. El modelo de isoterma de Langmuir se ajusta de manera más apropiada a estos resultados. Se encontró que el modelo cinético de pseudo segundo orden describe muy bien el proceso de adsorción del colorante rojo allura con los carbones activados.
\end{abstract}

Palabras clave: carbón activado, rojo allura, isotermas de Langmuir, modelo cinético, isotermas de nitrógeno

\section{Allura Red Dye Removal from Aqueous Solutions using Activated Carbons obtained from Agricultural Waste}

\begin{abstract}
The removal of allura red dye with different type of activated carbon obtained from vegetable waste was studied. The carbons were characterized by infrared spectroscopy, X-ray diffraction and electron microscopy obtaining typical results for carbonaceous materials. The surface areas of activated carbon were determined by nitrogen isotherms. Similarly, dye removal isotherms were evaluated taking into account, contact time effect, $\mathrm{pH}$, initial concentration and amount of adsorbent. Corn cob carbons, showed higher removal percentage with respect to the carbons of coconut husks and of rice husks. Langmuir's isotherm model showed better correlation of these results. It was found that a pseudo second order kinetics model well describes the adsorption of allura red dye with activated carbons.
\end{abstract}

Keywords: activated carbon, allura red, Langmuir's isotherm, kinetics model, nitrogen isotherms 


\section{INTRODUCCIÓN}

Los análisis fisicoquímicos de aguas residuales provenientes de la industria textil, cuero, cosméticos, papel, impresión, plásticos, productos farmacéuticos, alimentos, entre otros, han mostrado que contienen concentraciones significativas de colorantes especialmente del tipo azo, lo cual ha generado una problemática ambiental (Chiou et al.,2004). La eliminación de estos colorantes de las aguas residuales industriales se ha convertido en uno de los principales problemas para las industrias, debido a que los colorantes son moléculas orgánicas recalcitrantes, resistentes a la digestión aeróbica, y son estables a la luz, calor y agentes oxidantes por consiguiente difíciles de tratar (Kamal, 2009). En consecuencia, actualmente existe gran interés mundial por encontrar métodos eficientes y económicos para el tratamiento de agua. Así por ejemplo, en la literatura científica se reporta el uso de varios métodos físicos y químicos para la remoción de colorantes presentes en aguas residuales, entre los que se encuentra la degradación microbiana aeróbica y anaeróbica, la coagulación, la oxidación química, procesos de separación de membrana, electroquímica, dilución, filtración, osmosis reversa, entre otros (Singh et al., 2003). No obstante, estas metodologías resultan costosas al momento de su aplicación por lo que se requiere la búsqueda de otras alternativas que sean eficientes y económicas atractivas.

En este sentido, el carbón activado puede ser una alternativa en el tratamiento de aguas residuales, debido a que estos materiales presentan grandes áreas superficiales, elevado poder de adsorción y estructuras porosas (Wojciech et al., 2013). En la actualidad existen investigaciones en donde se utiliza el carbón activado para la remoción de contaminantes en aguas residuales. Así, Robinson y colaboradores (Robinson et al., 2002; Han et al., 2007; Wang et al., 2010), reportaron que los carbones activos provenientes de residuos agrícolas tales como cáscara de mazorca de maíz y cebada, presentaron eficientes propiedades adsorbentes de colorantes textiles en efluentes industriales (Khaled et al., 2009). Asimismo, McKay y colaboradores (McKay et al.,1986) estudiaron la adsorción de los colorantes azoicos rojo allura y amarillo anaranjado a partir de soluciones acuosas sobre carbón activo, dando idea del tipo de interacciones adsorbato-adsorbente que pueden ocurrir en estos sistemas.

Por lo anterior, el objetivo que persiguió este trabajo fue obtener diferentes tipos de carbón activado a partir de desechos agrícolas tales como cáscara de coco, cascarilla de arroz y tuza de maíz para su estudio fisicoquímico, con el fin de determinar su utilidad en la remoción del colorante azoico rojo allura (figura 1), el cual es ampliamente usando en la industria de alimentos y textil. También, se evaluó la cinética de adsorción de rojo allura en los diferentes tipos de carbón activado, determinando la velocidad de remoción del colorante y el tiempo de residencia en la interface solución-adsorbente.

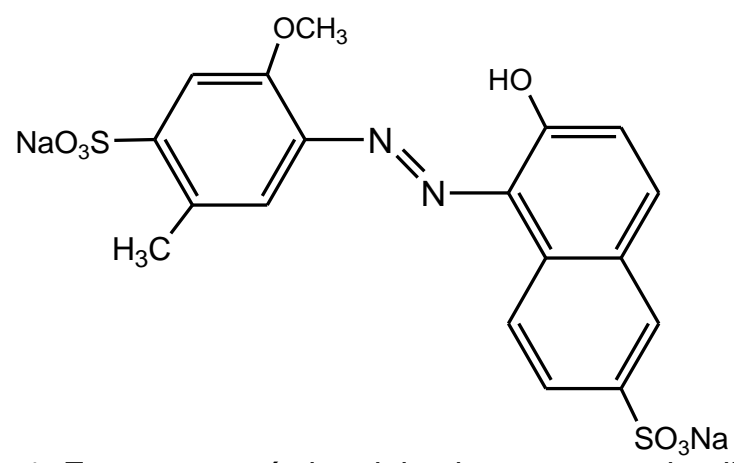

Fig. 1: Estructura química del colorante azo rojo allura

\section{METODOLOGÍA}

Los carbones utilizados fueron obtenidos a partir de desechos agrícolas: cáscara de coco, cascarilla de arroz y tuza de maíz, en el Departamento de Córdoba-Colombia. Estos materiales se caracterizaron mediante espectroscopia infrarroja (Zhao et al., 2009a; Zhao et al., 2009b), difracción de rayos X (Huang et al., 2011) y microscopía electrónica de barrido. Las áreas superficiales de los carbones activados se determinaron a partir de las isotermas de nitrógeno (Sun et al., 2012; Mopoung et al., 2008). El colorante rojo allura (C.I. 16035, código FD\&C Red N40, $\lambda_{\operatorname{máx}}=504 \mathrm{~nm}$ ) se obtuvo de Sigma Chemical Company.

Las soluciones del colorante rojo allura se prepararon midiendo una cantidad exacta del colorante disuelto en agua destilada, para preparar las soluciones madre de concentración $1 \mathrm{~g} / \mathrm{L}$, posteriormente se prepararon las soluciones estudio de concentración que osciló entre $2 \mathrm{mg} / \mathrm{L}$ hasta $100 \mathrm{mg} / \mathrm{L}$. La cantidad del colorante removido se determinó mediante la búsqueda de la absorbancia en la longitud de onda característica del colorante, utilizando un Espectrofotómetro UV-Vis ThermoScientific Serie GENESYS 10S. Para determinar 
el efecto de la incidencia de los parámetros: tiempo de contacto (t), $\mathrm{pH}$, cantidad de adsorbente (m) y concentración inicial $\left(\mathrm{C}_{0}\right)$ en la remoción del colorante rojo allura, se realizaron los experimentos a temperatura ambiente. En cada experimento se pusieron en contacto $10 \mathrm{~mL}$ de la solución de colorante (de concentración y pH deseado) con una cantidad conocida de carbón activado en un matraz de $100 \mathrm{~mL}$, esta mezcla se agitó a temperatura ambiente en un sheker a una velocidad constante de 150 rpm.

\section{RESULTADOS Y DISCUSIÓN}

\section{Difracción de Rayos $X$}

El análisis estructural de los tres carbones utilizados, carbón cáscara de coco (CCC), cascarilla de arroz (CCA) y tuza de maíz (CCTM), se realizó por difracción de rayos $X$, la cual mostró perfiles típicos de materiales carbonosos (figura 2) (Zhao et al., 2009a; Zhao et al., 2009b), con características bastante amorfas con señales anchas y de poca intensidad. Sólo el carbón tuza de maíz activado (CCTMA) o sin activar (CCTMSA), presenta señales intensas, propias de componentes cristalinos dentro del material. Se

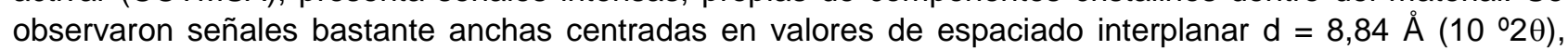

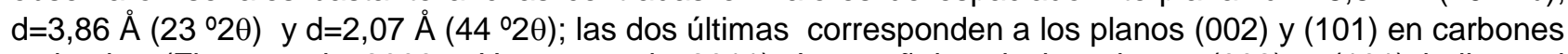
activados (Zhao et al., 2009a; Huang et al., 2011). Las señales de los planos (002) y (101) indican el apilamiento desordenado de microestructuras grafíticas o similares al grafito dentro de los materiales. Para

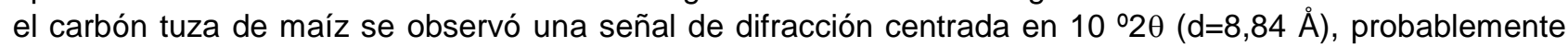
corresponda a la conformación de microestructuras laminares en los materiales carbonáceos, esta señal intensa indica cristalinidad en el carbón, probablemente originada de materiales lignocelulósicos con cierto agrupamiento estructural (Barpanda et al., 2011; Ryu et al., 2002).

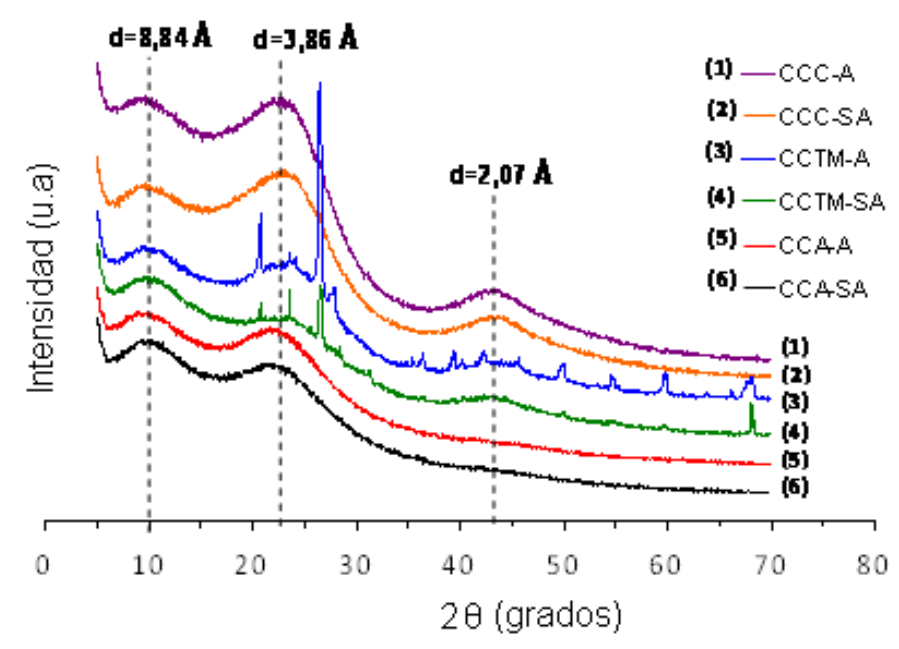

Fig. 2: Perfiles de difracción de rayos $X$ para los materiales carbonáceos activados (A) y sin activar (SA) obtenidos a partir de diferentes desechos agrícolas

\section{Espectroscopia Infrarroja (IR)}

Las características químicas de los carbones activos están determinadas en gran medida por un cierto grado de heterogeneidad química de la superficie por la presencia de heteroátomos tales como; oxígeno, nitrógeno, hidrógeno, azufre y fósforo (Shafeeyan et al., 2010). El análisis de los diferentes grupos funcionales existentes en la superficie de cualquier sólido es importante para diferentes aplicaciones como adsorción, catálisis y reactividad del sólido en general. Los espectros IR de los carbones obtenidos (figura 3), mostraron bandas con vibraciones similares en todos los casos, típicas de grupos funcionales presentes en el material celulósico transformado. Dado que las muestras fueron secadas previamente a $60^{\circ} \mathrm{C}$ durante 24 horas, la señal alrededor de $3430 \mathrm{~cm}^{-1}$ puede asignarse casi en su totalidad a vibraciones de estiramiento (tensión) de los grupos O-H formando puentes de hidrógeno (Huang et al., 2011; Boonamnuayvitaya et al., 2005). Las señales observadas alrededor de 2920 y $2850 \mathrm{~cm}^{-1}$ se atribuyen a vibraciones simétricas y asimétricas (Huang et al., 2011; Boonamnuayvitaya et al., 2005), de estiramiento de grupos - $\mathrm{CH}_{2}-$. El pico situado alrededor de $1560 \mathrm{~cm}^{-1}$ corresponde a las vibraciones de grupos $\mathrm{C}=\mathrm{C}$ en los anillos aromáticos de los materiales debido a la carbonización. El pico a $1613 \mathrm{~cm}^{-1}$ corresponde a grupos $\mathrm{C}=\mathrm{O}$, muy marcados en CCTM y la señal cercana a $1100 \mathrm{~cm}^{-1}$ es asignada a vibraciones de grupos C-O- en la misma región de SiO, además, esta banda está acompañada de una señal de entre 400 y $500 \mathrm{~cm}^{-1}$, que se atribuye a las vibraciones de los enlaces $\mathrm{Si}$ - O en sílice $\left(\mathrm{SiO}_{2}\right.$ ) descansa ( Zhu et al. , 2012; Zhao et al., 2009a; Huang et 
al., 2011; Boonamnuayvitaya et al., 2005). Debe tenerse en cuenta que, la señal a $3430 \mathrm{~cm}^{-1}$ es bastante ancha y podría involucrar también vibraciones de tensión de grupos $\mathrm{O}-\mathrm{H}$ de grupos carboxílicos. La vibración en $1384 \mathrm{~cm}^{-1}$ corresponde a flexiones de grupos $-\mathrm{CH}_{2}$ - Finalmente, los picos observados en 875 y $800 \mathrm{~cm}^{-1}$ se originan debido a vibraciones de enlace $\mathrm{C}-\mathrm{H}$ en los anillos aromáticos de los carbones (Zhao et al., 2009a; Asadullah et al., 2010).

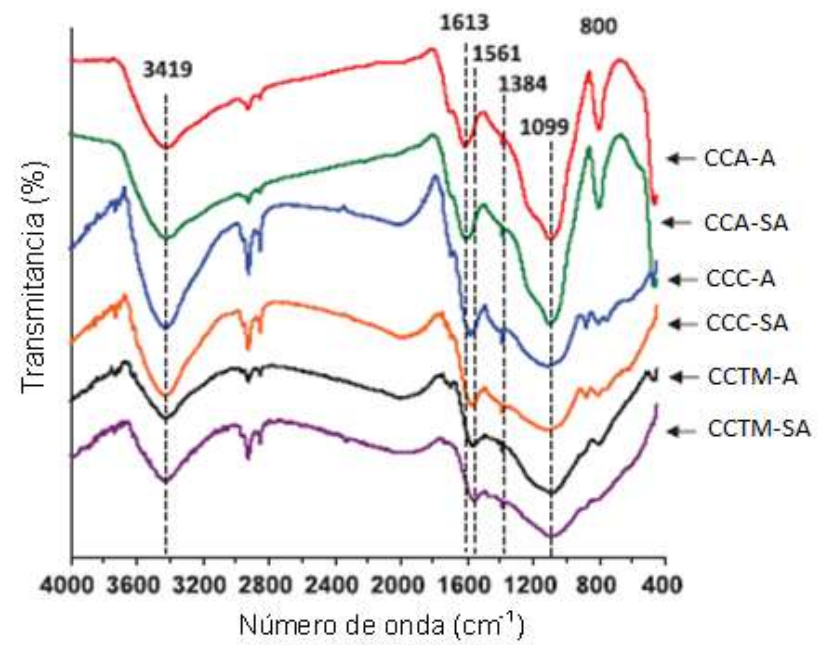

Fig. 3: Espectros IR de los carbones activados y sin activar obtenidos a partir de diferentes desechos agrícolas

\section{Adsorción de Nitrógeno}

En la figura 4, se muestran las isotermas de nitrógeno para los diferentes carbones preparados a partir de desechos agrícolas. Todas las isotermas de adsorción de nitrógeno fueron tipo I según la clasificación de la IUPAC (Leofanti, 1998; Stoeckli, 2005), y son típicas de materiales microporosos (Gómez-Serrano et al., 2001). En general, todos los carbones preparados mostraron altos niveles de adsorción, especialmente el obtenido a partir de cáscara de coco (CCC). En la tabla 1, se presentan los valores de área superficial obtenidos para los materiales cabonáceos preparados. De la tabla 1, es evidente que los valores determinados por el modelo de Langmuir son más altos que los cálculos por el modelo BET, lo cual básicamente se debe a la naturaleza microporosa de estos materiales.

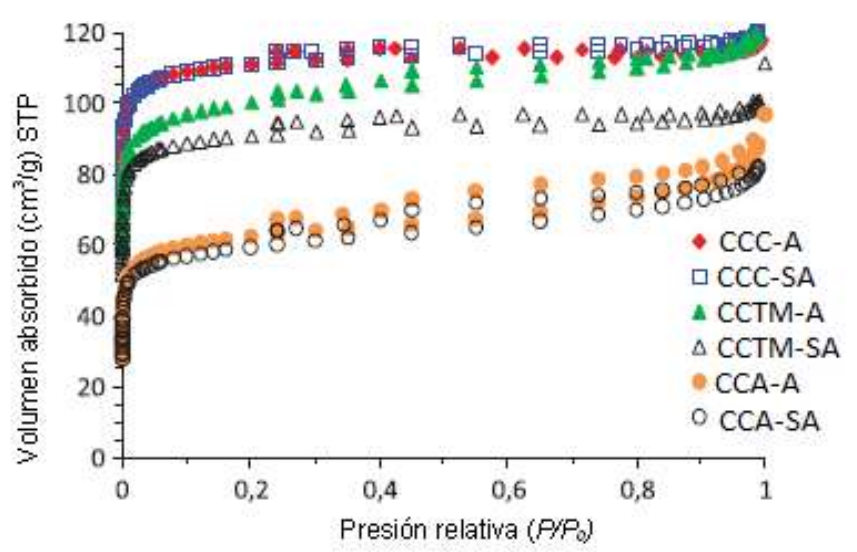

Fig. 4: Isotermas de adsorción de Nitrógeno a 77K de los carbones preparados a partir de los diferentes desechos agrícolas

Los valores de área superficial de CCC son más altos que para el resto de materiales, lo cual está en buen acuerdo con los niveles de adsorción de nitrógeno descritos por las isotermas (figura 4). Además, como resultado del proceso de activación (CCC-A, CCTM-A y CCA-A, respectivamente) se observa un pequeño incremento en los valores de área superficial. Adicionalmente, en la tabla 1, se presentan altas áreas de microporo, confirmando con ello la naturaleza microporosa de estos materiales. Asimismo, se calcularon los volúmenes totales de poro para todos los carbones, encontrándose altos volúmenes totales de poro para CCC y CCTM respectivamente. 
Tabla 1: Área superficial, área de poro y volumen total de poro para los carbones activados (A) y sin activar (SA)

\begin{tabular}{|l|c|c|c|c|}
\hline \multicolumn{1}{|c|}{ Material } & Área BET $\left(\mathrm{m}^{2} / \mathrm{g}\right)$ & Área Langmuir $\left(\mathrm{m}^{2} / \mathrm{g}\right)$ & Área de microporo $\left(\mathrm{m}^{2} / \mathrm{g}\right)$ & Volumen total de poro $\left(\mathrm{cm}^{3} / \mathrm{g}\right)$ \\
\hline CCC-SA & 334 & 458 & 286 & 0,1829 \\
\hline CCC-A & 334 & 458 & 283 & 0,1853 \\
\hline CCTM-SA & 306 & 403 & 230 & 0,1894 \\
\hline CCTM-A & 274 & 373 & 228 & 0,1726 \\
\hline CCA-SA & 192 & 245 & 135 & 0,1499 \\
\hline CCA-A & 183 & 234 & 127 & 0,1448 \\
\hline
\end{tabular}

\section{Microscopía Electrónica de Barrido}

El análisis por microscopía electrónica proporciona información sobre la morfología de los sólidos, lo cual es fundamental para comprender el desempeño de los materiales en diferentes aplicaciones (Huang et al., 2011). La morfología variada de estos carbones preparados a partir de diferentes materiales vegetales de desechos se observó su variación en el tamaño de partícula, que en general oscila entre 20 y $500 \mu \mathrm{m}$. En todas las partículas se observaron cavidades u oquedades de diferentes magnitudes, las cuales podrían contribuir al aumento del área superficial de los sólidos y cumplir con las funciones importantes en los procesos de adsorción de moléculas (figura 5).

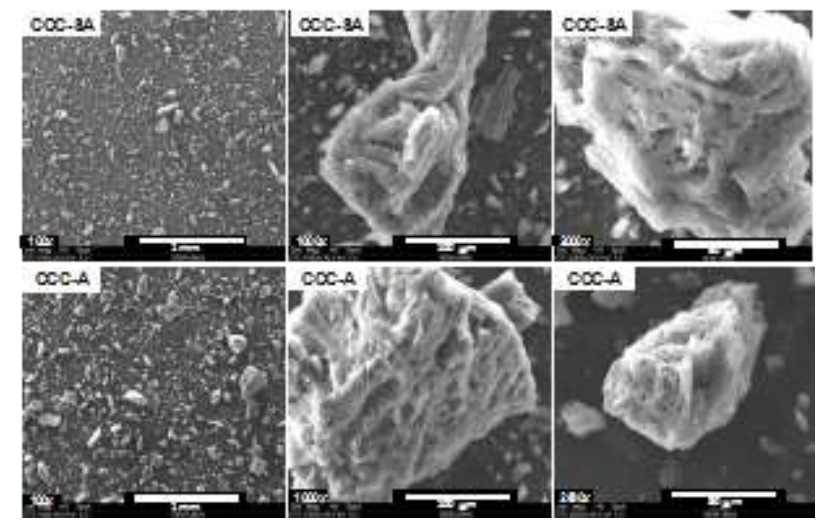

(a)

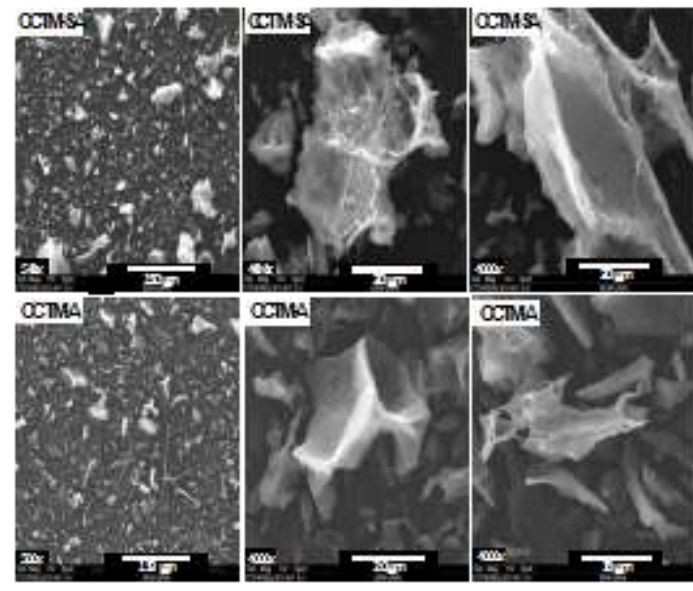

(b)

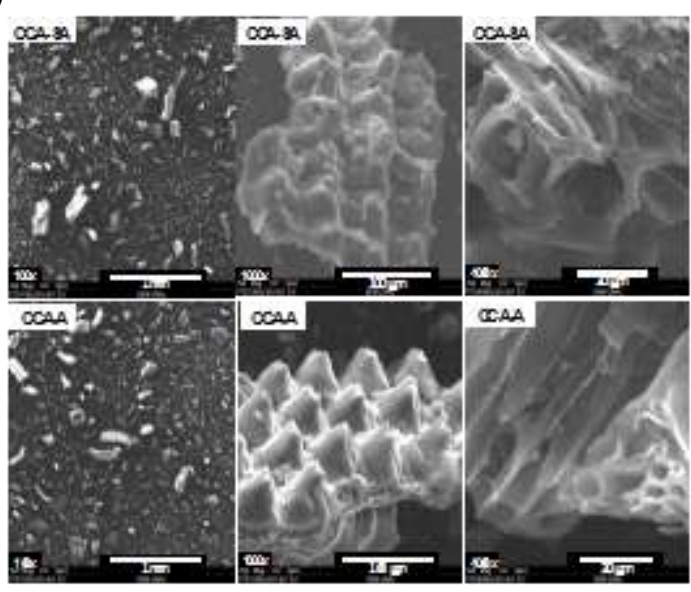

(c)

Fig. 5: a) micrografía SEM para CCC-SA y CCC-A; b) micrografía SEM para CCTM-SA y CCTM$A ; c)$ micrografía SEM para CCA-SA y CCA-A

La formación de estas cavidades puede estar relacionada con la expulsión de gases o vapores y compuestos volátiles durante los procesos de calcinación, como ya se ha indicado en literatura (Sun et al., 2012; Tan et al., 2008). Las partículas de los carbones CCC, muestran una morfología más compacta que el resto de materiales. Las partículas de carbón procedentes de la carbonización de CCTM poseen morfología bastante laminar en forma de hojuelas; mientras que los carbones obtenidos de cascarilla de arroz muestran superficies bastante rugosas, como ya lo habían observado otros investigadores para la cascarilla de arroz calcinada (Asadullah et al., 2010). 


\section{Adsorción de Rojo Allura}

Para el estudio de la adsorción del colorante rojo allura sobre carbón activado, se tuvieron en cuenta los siguientes parámetros: el tiempo de contacto, $\mathrm{pH}$ de la solución, cantidad de adsorbente y concentración inicial de la solución (Doig et al., 2011; Yahya et al.; 2008). Las condiciones para determinar los efectos de estos parámetros fueron: $\mathrm{pH}=7$, cantidad de carbón activado de $0,2 \mathrm{~g}$, una concentración de colorante rojo allura de $10 \mathrm{mg} / \mathrm{L}$ para el CCA y CCC respectivamente, y para CCTM una concentración de colorante de $70 \mathrm{mg} / \mathrm{L}$ debido a su mayor capacidad de adsorción.

\section{Efecto del Tiempo de contacto}

El efecto del tiempo de contacto para la remoción del colorante rojo allura mostró una rápida adsorción por parte de los diferentes tipos de carbón en las cinco primeras horas, como se observa en la figura 5a. Posteriormente, los carbones siguen adsorbiendo, pero la relación de la cantidad de colorante adsorbido se mantiene constante. Asimismo, se observó que la máxima adsorción del colorante rojo allura se logró con CCTM, en una solución de concentración de $70 \mathrm{mg} / \mathrm{L}$, logrando una remoción de aproximadamente un $97 \%$ del colorante en 5 horas. Para los carbones CCC y CCA la concentración utilizada fue menor (10mg/L), obteniéndose porcentajes de remoción de 85 y 57\% respectivamente. Estos resultados están acordes con las estructuras morfológicas y las áreas microporosas de los carbones utilizados. El carbón proveniente de la tuza de maíz, mostró mayor capacidad de adsorción para este colorante, lo cual se puede atribuir a la porosidad de este material y la afinidad química entre la superficie de CCTM y el colorante. Asimismo, el carbón obtenido de cáscara de coco muestra una superficie morfológica mas compacta y presenta mayor área de microporo, que CCTM y CCA (Zhu et al., 2012). Las partículas de los carbones obtenidos de cascarilla de arroz aparentan una morfología bastante rugosa y menor área de microporo que los otros carbones (tabla 1).

\section{Efecto del $\mathrm{pH}$}

El pH es otro factor determinante en la adsorción de colorantes en solución acuosa (Aksu et al., 2005), debido a que el pH afecta la estructura del colorante (Crini et al., 2007). Este parámetro no solo afecta la carga del colorante sino el grado de ionización del mismo y además puede provocar una disminución de color en la solución (Aksu et al., 2005). El estudio del efecto del pH en la adsorción del azocolorante rojo allura, se realizó sobre los tres tipos de carbón activado (CCC, CCA y CCTM), en el rango de pH de 3 a 10. Los resultados obtenidos de la adsorción del azocolorante, se muestran en la figura $5 \mathrm{~b}$, e indican que a $\mathrm{pH}$ ácido (3 y 5), se adsorbe aproximadamente un 95\% del colorante, siendo la mayor adsorción a pH 3; mientras que a pH básico, la adsorción disminuye aproximadamente en un $25 \%$. También, se observa en la figura $5 \mathrm{~b}$ que CCTM continua siendo el material carbonáceo de mayor adsorción.

El colorante en solución acuosa se disocia formando el anión sulfito del colorante y los respectivos iones sodio $\left(\mathrm{Na}^{+}\right)$(McKay et al., 1986). A pH ácido, la superficie del adsorbente se carga positivamente, debido a la alta concentración de iones $\mathrm{H}^{+}$en el medio; por tal motivo hay mayor atracción electrostática entre la carga positiva en la superficie del carbón y la carga negativa del anión del azocolorante (Ribeiro et al., 2012; Goel et al., 2005; Crini et al., 2007). La disminución de la capacidad de adsorción a pH básico, se relaciona principalmente con el incremento de la carga superficial negativa del carbón, por lo que podría producirse una repulsión electrostática con los aniones del colorante.

\section{Efecto de la concentración inicial}

Otro parámetro estudiado fue el efecto de la concentración inicial en la remoción del colorante rojo allura, por medio de carbones activos CCC, CCA y CCTM, cuya importancia radica en que la determinación de este parámetro mide la resistencia de la adsorción entre la solución acuosa del colorante y la fase sólida del carbón (Aksu, 2005). En la figura 6c, se muestran las concentraciones iniciales utilizadas para los tres tipos de carbones activos, y se observa que la máxima adsorción corresponde a CCTM, seguido por CCC y CCA respectivamente. Los porcentajes de remoción obtenidos para el colorante rojo allura con CCTM, muestra un porcentaje de remoción casi constante en todo el rango de concentraciones iníciales estudiadas. Para los carbones CCC y CCA, el máximo porcentaje de remoción se presenta a una concentración inicial de 10 $\mathrm{mg} / \mathrm{L}$, a partir del cual se mantiene constante. Estos resultados indican que el cambio en la concentración afecta la tasa de saturación y tiempo de penetración en estos carbones (Wang et al., 2010). CCTM, por tener mayor capacidad de adsorción se afecta en menor grado que los otros dos carbones. En la figura 6c, es evidente que la capacidad de adsorción de los carbones CCC y CCA, es menor que en CCTM lo cual se atribuye a la poca capacidad de adsorción de estos carbones debido a su estructura morfológica (Chiang et al., 2007). 
a.

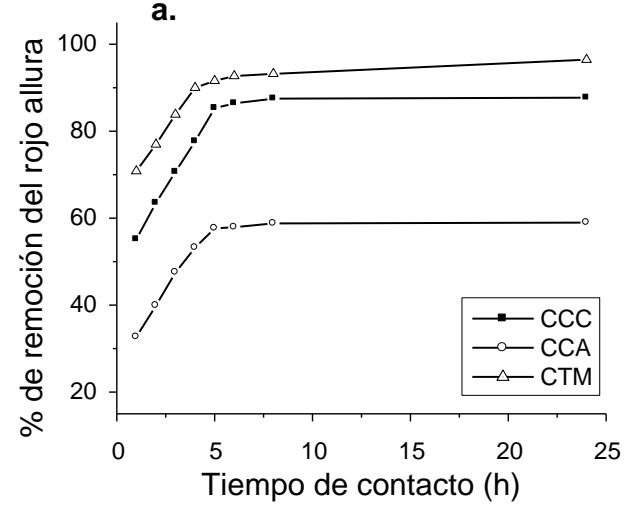

c.

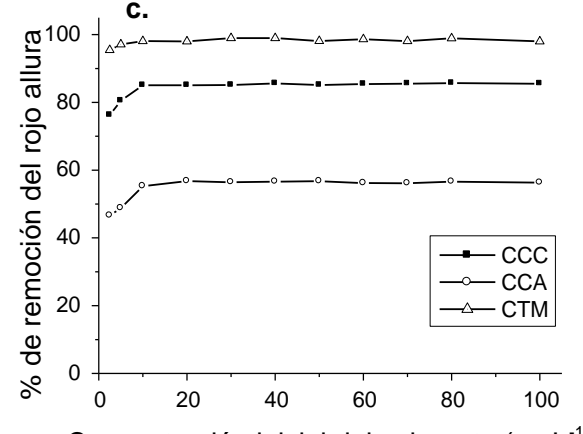

Concentración inicial del colorante $\left(\mathrm{mgL}^{-1}\right)$

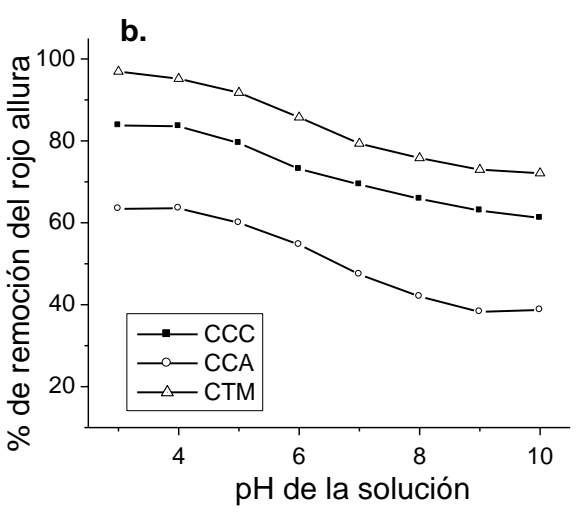

d.

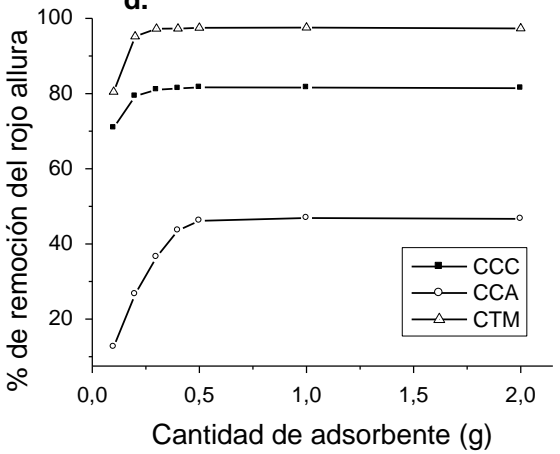

Fig. 6: Isotermas de adsorción del colorante rojo allura medidos a diferentes parámetros: (a) tiempo de contacto; (b) $\mathrm{pH}$; (c) concentración inicial del colorante y (d) cantidad de adsorbente

\section{Efecto de la cantidad de carbón}

El efecto de la cantidad de adsorbente en la remoción del colorante rojo allura, se estudió en un rango de 0,1 a $2,0 \mathrm{~g}$ de carbón. En la figura $6 \mathrm{~d}$, se observa un aumento gradual del porcentaje de remoción del colorante con el incremento de la cantidad de carbón, debido a que se proporciona mayor área de superficie del adsorbente y disponibilidad de más sitios de adsorción. Sin embargo, existe una cantidad límite de carbón, donde el porcentaje de remoción permanece contante (Zhu et al., 2012). La cantidad óptima de CCTM para la remoción del colorante rojo allura a partir de una solución de concentración de $70 \mathrm{mg} / \mathrm{L}$, fue de $0,3 \mathrm{~g}$. La remoción del azocolorante fue aproximadamente de 97\%. Por lo tanto, CCTM remueve aproximadamente $226,33 \mathrm{mg}$ de colorante por cada gramo de carbón $(\mathrm{mg} / \mathrm{g})$. El alto porcentaje de remoción obtenido para CCTM, muestra la excelente capacidad de adsorción de este material carbonáceo, lo cual hace factible proponer su aplicabilidad potencial en la fabricación de filtros para la remoción de este tipo de colorantes.

\section{Isotermas de adsorción}

Las isotermas de adsorción se determinaron para la distribución del adsorbente y el adsorbato en un sistema sólido-líquido. Las isotermas de Freudlich y Langmuir son los modelos más utilizados para explicar el comportamiento de adsorción de estos sistemas (Sun et al., 2012; loannidou et al., 2010). Como se mostró en la tabla 1, el proceso de remoción del colorante rojo allura se ajusta al modelo Langmuir (Wang et al., 2010; Gómez-Serrano, 2001). Para determinar el mejor modelo de isoterma de adsorción, se tomó como criterio de selección la regresión lineal comparando los coeficientes de regresión obtenidos en cada caso (Khaled et al., 2009); siendo Langmuir el mejor modelo. La isoterma Langmuir esta descrita por la ecuación:

$$
\mathrm{Q}_{\mathrm{eq}}=\frac{\mathrm{Q}_{\max } \mathrm{bC}_{\mathrm{eq}}}{1+\mathrm{bC}_{\mathrm{eq}}}
$$

Donde, $Q_{\max }$ y b son los parámetros de Langmuir relacionados a la máxima capacidad de adsorción y la energía de enlace de la adsorción respectivamente. Qeq, es la concentración del soluto retenido en el adsorbente en el equilibrio $(\mathrm{mg} / \mathrm{g})$. $\mathrm{C}_{\text {eq, }}$, es la concentración de soluto en la solución en el equilibrio (mg/L). 
En la tabla 2, se muestran los parámetros obtenidos cuando se describe el proceso de adsorción mediante las isotermas de Langmuir.

Tabla 2: Parámetros de Langmuir para rojo allura

\begin{tabular}{|c|r|r|r|}
\hline Parámetros & \multicolumn{1}{c|}{ CCC } & \multicolumn{1}{c|}{ CCA } & \multicolumn{1}{c|}{ CCTM } \\
\hline$Q_{\max }$ & 1102,530 & 1907,341 & 4070,501 \\
\hline $\mathrm{b}$ & 0,193 & 0,012 & 1,089 \\
\hline $\mathrm{R}^{2}$ & 0,992 & 0,999 & 0,996 \\
\hline
\end{tabular}
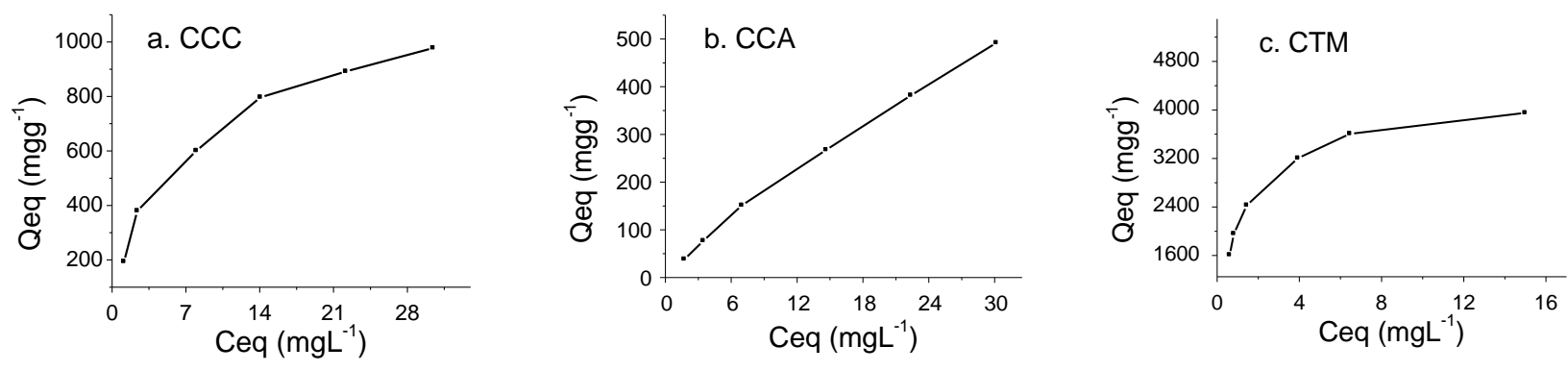

Fig. 7: Isotermas de adsorción de Langmuir para rojo allura para: (a) CCC, (b) CCA y (c) CCTM respectivamente.

\section{Cinética de la Adsorción}

El estudio cinético del proceso de adsorción permitió determinar la velocidad de adsorción del colorante rojo allura de la fase acuosa. Se probaron varios modelos cinéticos para describir el proceso de acuerdo con los diferentes parámetros medidos en las isotermas de adsorción. De acuerdo, con los datos cinéticos presentados en la tabla 3, se observa que la cinética adsorción se ajusta a un modelo cinético de pseudosegundo orden, ya que, presenta mayores valores de $\mathrm{R}^{2}$. El modelo cinético de pseudo-segundo orden se expresa como:

$$
\frac{\mathrm{t}}{\mathrm{q}_{\mathrm{t}}}=\frac{1}{\mathrm{~K}_{2} \mathrm{q}_{\mathrm{e}}^{2}}+\frac{1}{\mathrm{q}_{\mathrm{e}}} \mathrm{t}
$$

Donde: $k_{2}$ es la constante de velocidad de adsorción, $q_{e}$ es la cantidad de colorante adsorbida en el equilibrio $(\mathrm{mg} / \mathrm{g})$ y $\mathrm{q}_{\mathrm{t}}$ es la cantidad de colorante adsorbido en el tiempo $\mathrm{t}(\mathrm{mg} / \mathrm{g})$. La capacidad de equilibrio de adsorción $\left(\mathrm{q}_{\mathrm{e}}\right)$ y la constante de velocidad de segundo orden $\left(\mathrm{k}_{2}\right)$ ( $\mathrm{g} / \mathrm{mgmin}$ ) puede determinarse experimentalmente a partir de la pendiente y la intersección en el gráfico de t /q versus t.

En la tabla 3 , se presentan las constantes de velocidad, capacidad de equilibrio y $\mathrm{R}^{2}$ para los diferentes carbones investigados.

Tabla 3: Parámetros cinéticos de los modelos de pseudo-primer y segundo orden para rojo allura con los carbones activos estudiados. Unidades de qe (mg de colorante/g de carbón activado)

\begin{tabular}{|c|c|r|c|c|c|c|}
\hline \multirow{2}{*}{ Tipo de carbón } & \multicolumn{3}{|c|}{ Modelo cinético pseudo-primer orden } & \multicolumn{3}{c|}{ Modelo cinético pseudo-segundo orden } \\
\cline { 2 - 7 } & $\mathrm{K}_{1}\left(\mathrm{~min}^{-1}\right)$ & $\mathrm{q}_{\mathrm{e}}\left(\mathrm{mgg}^{-1}\right)$ & $\mathrm{R}^{2}$ & $\mathrm{~K}_{2}\left(\mathrm{gmg}^{-1} \mathrm{~min}^{-1}\right)$ & $\mathrm{q}_{\mathrm{e}}\left(\mathrm{mgg}^{-1}\right)$ & $\mathrm{R}^{2}$ \\
\hline $\mathrm{CCC}$ & $4,353^{*} 10^{-3}$ & 221,156 & 0,805 & 12,240 & 6,136 & 0,988 \\
\hline CCA & $8,735^{*} 10^{-4}$ & 172,186 & 0,839 & 7,424 & 4,967 & 0,996 \\
\hline CTM & $2,610^{*} 10^{-2}$ & 7,557 & 0,751 & 36,363 & 3,817 & 0,987 \\
\hline
\end{tabular}

En la figura 8, se muestran las gráficas de t/q vs. t para los diferentes carbones investigados. Los resultados de la cinética de adsorción obtenidos en este trabajo están en buen acuerdo con reportes experimentales previos para carbones activados, donde se ha mostrado que la cinética se ajusta a un modelo de pseudosegundo orden (Wang et al., 2010; Dogan et al., 2009). 

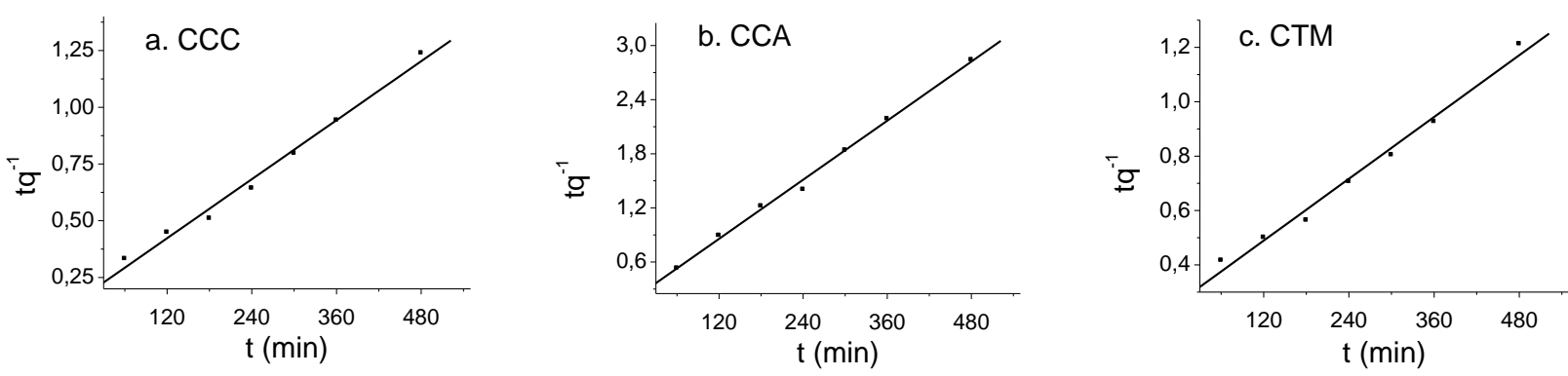

Fig. 8: Modelo cinético de pseudo-segundo orden para rojo allura con CCC, CCA y CCTM respectivamente

\section{CONCLUSIONES}

Los resultados de la caracterización de los carbones, mostraron que se obtuvieron carbones activos con áreas superficiales de $458 \mathrm{~m}^{2} / \mathrm{g}$ para carbón cáscara de coco, $373 \mathrm{~m} 2 / \mathrm{g}$ para el carbón tuza de maíz y 234 $\mathrm{m}^{2} / \mathrm{g}$ para carbón cascarilla de arroz y unas áreas de microporo de $283 \mathrm{~m}^{2} / \mathrm{g}, 228 \mathrm{~m}^{2} / \mathrm{g} \mathrm{y} 127 \mathrm{~m}^{2} / \mathrm{g}$ respectivamente.

Se encontró que el carbón obtenido de tuza de maíz (CCTM) tiene una alta capacidad de adsorción del azocolorante rojo allura como lo indicaron los porcentajes de remoción. El carbón obtenido de cáscara de coco presentó niveles de adsorción buenos, debido a que presenta una típica adsorción física donde prima su mayor área de microporo y el carbón de cascarilla de arroz no presentó buenas características como material adsorbente para este colorante, ya que presenta menor área de microporo.

Para los tres tipos de carbones utilizados en la remoción del colorante rojo allura, el modelo cinético que mejor explicó el proceso de adsorción fue el de pseudo-segundo orden, y las isotermas de adsorción se ajustaron mejor al modelo de Langmuir. La adsorción del azocolorante con carbón activado mostró una sensibilidad a la concentración inicial, el tiempo de contacto, el pH de la solución y la cantidad de adsorbente.

\section{AGRADECIMIENTOS}

Los autores de este artículo muestran su agradecimiento al Centro de Investigaciones Universitarias (CIUC), de la Universidad de Córdoba, por el apoyo económico brindado (proyecto: código 1.2.08.109 y numeral FCB-03-09). Al Dr. José Gregorio Carriazo, de la Universidad Nacional de Colombia.

\section{REFERENCIAS}

Aksu, Z., Application of biosorption for the removal of organic pollutants: a review, Process Biochem: 40, 9971026 (2005).

Asadullah M. y otros cuatro autores, Chemical and structural evaluation of activated carbon prepared from jute sticks for Brilliant Green dye removal from aqueous solution, J. Hazard. Mater: 174, 437-443 (2010).

Barpanda, P., G. Fanchini y G.G., Amatucci, Structure, surface morphology and electrochemical properties of brominated activated carbons, Carbon: 49, 2538-2548 (2011).

Boonamnuayvitaya, V, S. Sae-ung y W. Tanthapanichakoon, Preparation of activated carbons from coffee residue for the adsorption of formaldehyde, Sep. Purif. Technol: 42,159-168 (2005).

Chiang Hung-Lung, y otros cuatro autores, Dye adsorption on biosolid adsorbents and commercially activated carbon, Dyes Pigments: 75, 52-59 (2007).

Chiou, M.S., P.Y. Ho y H.Y. Li, Adsorption of anionic dyes in acid solutions using chemically cross-linked chitosan beads, Dyes Pigments: 60, 69-84 (2004).

Crini, G., H.N. Peindy, F, Gimbert y C. Robert, Removal of C.I. Basic Green 4 (Malachite Green) from aqueous solutions by adsorption using cyclodextrin-based adsorbent: Kinetic and equilibrium studies, Sep. Purif. Technol: 53, 97-110 (2007).

Dogan, M., M.H. Karaoğlu y M. Alkan, Adsorption kinetics of maxilon yellow 4GL and maxilon red GRL dyes on kaolinite, J. Hazard. Mater: 165, 1142-1151 (2009).

Doig, P. y A. Lucía, Estudio de la adsorción de compuestos aromáticos mediante carbón activado preparado a partir de la cáscara de castaña, Tesis de Doctorado, Facultad de Ciencias e Ingeniería, Pontificia Universidad Católica del Perú , Perú, 2011. 
Goel, J., K. Kadirvelu, C. Rajagopal y V.K. Garg, Removal of Lead(II) from Aqueous Solution by Adsorption on Carbon Aerogel Using a Response Surface Methodological Approach, Ind. Eng. Chem. Res: 44, 1987-1994 (2005).

Gómez-Serrano, V., C. González-García y M. González-Martín, Nitrogen adsorption isotherms on carbonaceous materials. Comparison of BET and Langmuir surface areas, Powder Technol: 116, 103-108 (2001).

Huang, L., Sun, Y., Wang, W., Yue, Q. y Yang, T. Comparative study on characterization of activated carbons prepared by microwave and conventional heating methods and application in removal of oxytetracycline (OTC), Chem. Eng. J.: 171, 1446-1453 (2011).

Ioannidou, O.A. y otros cuatro autores, Preparation of activated carbons from agricultural residues for pesticide adsorption, Chemosphere: 80, 1328-1336 (2010).

Kamal A., Removal of direct blue-106 dye from aqueous solution using new activated carbons developed from pomegranate peel: Adsorption equilibrium and kinetics, J. Hazard. Mater: 165, 52-62 (2009).

Khaled, A., A.E. Nemr, A. El-Sikaily y O. Abdelwahab, Removal of Direct N Blue-106 from artificial textile dye effluent using activated carbon from orange peel: Adsorption isotherm and kinetic studies, J.Hazard. Mater: 165, 100-110 (2009).

Leofanti, G., M. Padovan, G. Tozzola y B. Venturelli, Surface area and pore texture of catalysts, Catal. Today: 41, 207-219 (1998).

McKay, G., G. Ramprasad y P. Pratapa Mowl, Equilibrium studies for the adsorption of dyestuffs from aqueous solutions by low-cost materials. Water Air Soil Poll: 29, 273-283 (1986).

Mopoung, S, Surface Image of Charcoal and Activated Charcoal from Banana Peel, J. Microsc. Soc. Thailand: 22 , 15-19 (2008).

Ribeiro, R., y otros cinco autores, Activated carbón xerogels for the removal of the anionic azo dyes Orange II and Chromotrope 2R byadsorption and catalytic wet peroxide oxidation, Chem. Eng. J: 195-196, 112-121 (2012).

Robinson, T., Removal of dyes from a synthetic textile dye effluent by biosorption on apple pomace and wheat straw, Water Res: 36, 2824-2830 (2002).

Ryu, Z., y otros cuatro autores, Microstructure and chemical analysis of PAN-based activated carbon fibers prepared by different activation methods, Carbon: 40, 1131-1150 (2002).

Shafeeyan, M.S., W. Daud, A. Houshmand, y A. Shamiri, A review on surface modification of activated carbon for carbon dioxide adsorption, J. Anal. Appl. Pyrrol: 89, 143-151 (2010).

Singh, K.P., y otros cuatro autores, Color Removal from Wastewater Using Low-Cost Activated Carbon Derived from Agricultural Waste Material, Ind. Eng. Chem. Res: 42, 1965-1976 (2003).

Stoeckli, F. y T.A. Centeno, On the determination of surface areas in activated carbons, Carbon: 43, 1184-1190 (2005).

Sun, Y. y otros cinco autores, Comparative study on characterization and adsorption properties of activated carbons with H3PO4 and H4P2O7 activation employing Cyperusalternifolius as precursor, Chem. Eng. J: 181182, 790-797 (2012).

Tan, I,A., A.L. Ahmad, y B.H. Hameed, Adsorption of basicdyeonhigh-surface-area activated carbón prepared from coconut husk: Equilibrium, kinetic and thermodynamic studies, J. Hazard. Mater: 154, 337-346 (2008).

Wang, L y otros cinco autores, Adsorption of basic dyes on activated carbon prepared from Polygonumorientale Linn: Equilibrium, kinetic and thermodynamic studies, Desalination: 254, 68-74 (2010).

Wojciech K.A. y otros cuatro autores, Application of hollow mesoporous carbon nanospheres as an high effective adsorbent for the fast removal of acid dyes from aqueous solutions, Chem. Eng. J: 228, 824-833 (2013).

Yahya, S. y I. Musa, El-Barghouthi, Amjad, H. El-Sheikh y Gavin, M. W., Effect of solution pH, ionic strength, and temperatura on adsorption behavior of reactive dyes on activated carbón, Dyes Pigments: 77, 16e, 23 (2008).

Zhao, J.G., F.Y. Li, y C.Q., Jin, Graphitization of activated carbon under high pressures and high temperatures, Solid State Commun: 149, 818-821 (2009).

Zhao,J y otros cuatro autores, Structural evolution in the graphitization process of activated carbon by highpressure sintering, Carbon: 47, 744-751 (2009).

Zhu, K., y otros cinco autores, Studies on removal of NH4+-N from aqueous solution by using the activated carbons derived from rice husk, Biomass and Bioenergy: 43, 18-25 (2012). 